\title{
INDEXAÇÃO DA REVISTA DE AGRICULTURA
}

OS ARTIGOS PUBLICADOS NA REVISTA DE AGRICULTURA SÃO RESUMIDOS NOS SEGUINTES PERIÓDICOS:

- ANIMAL BREEDING ABSTRACTS,

- BASE (Banco de Dados de Literatura Periódica em

Ciências Agrárias),

- BIOLOGICAL ABSTRACTS,

- CHEMICAL ABSTRACTS,

- CURRENT CROPS ABSTRACTS,

- FIELD CROPS ABSTRACTS,

- FOREST PRODUCTS ABSTRACTS,

- FORESTRY ABSTRACTS,

- HELMINTHOLOGICAL ABSTRACTS,

- HERBAGE ABSTRACTS,

- HORTICULTURAL ABSTRACTS,

- PERIODICA,

- PLANT BREEDING ABSTRACTS,

- REVIEW OF APPLIED ENTOMOLOGY,

- SOIL \& FERTILIZERS,

- TOBACCO ABSTRACTS,

- U.S. DEPARTMENT OF AGRICULTURE

- WEED ABSTRACTS,

- ZOOLOGICAL RECORD.

O PRESENTE FASCÍCULO FOI SUBVENCIONADO PELA FUNDAÇÃO DE ESTUDOS AGRÁRIOS LUIZ DE QUEIROZ (FEALQ). 